\title{
Impacto del concepto PLE en la literatura sobre educación: la última década
}

\section{(The PLE and its impact on the educational literature: the last decade)}

\author{
Linda Castañeda \\ Universidad de Murcia (España) \\ Gemma Tur \\ Universitat de les Illes Balears (España) \\ Ricardo Torres-Kompen \\ Universitat Ramón Llull - La Salle (España)
}

DOI: http://dx.doi.org/10.5944/ried.22.1.22079

\section{Como referenciar este artículo:}

Castañeda, L., Tur, G., y Torres-Kompen, R. (2019). Impacto del concepto PLE en la literatura sobre educación: la última década. RIED. Revista Iberoamericana de Educación a Distancia, 22(1), pp. 221-241. doi: http://dx.doi.org/10.5944/ ried.22.1.22079

\section{Resumen}

El concepto de Entorno Personal de Aprendizaje (PLE) es uno de los conceptos que ha recibido una mayor atención en los últimos años en el ámbito de la tecnología educativa. Los que estudiamos el tema asumimos - hay quien dice que de forma ingenua o acrítica- que se trata de un concepto realmente disruptivo, fundamental y que está en la base de los enfoques pedagógicos emergentes propios del momento tecno-social que nos enmarca, no solo en la faceta tecnológica-instrumental, sino también en los fundamentos mismos del proceso educativo. No obstante, poco sabemos sobre el impacto de este concepto y sus allegados en otros temas educativos no necesariamente ligados a la tecnología. A continuación, a través de una revisión de literatura sistematizada, se analiza el impacto del tema PLE en los tres principales focos temáticos de la literatura científica sobre educación de la última década: prácticas pedagógicas emergentes, aprendizaje autorregulado y desarrollo profesional docente. Se analiza si los PLE han impactado en cada uno de los temas; el nivel de importancia de ese impacto adquiere en cada uno de ellos y la forma en que se "materializa" en las diferentes temáticas. Las conclusiones de este artículo pretenden ofrecer sugerencias sobre cuál puede ser el futuro de la investigación y la práctica de un concepto que, tras algo más de diez años de su aparición en el ámbito educativo, sigue considerándose emergente. 
Palabras clave: entorno personal de aprendizaje; prácticas educativas emergentes; aprendizaje autorregulado; desarrollo profesional docente; revisión sistematizada de literatura.

\begin{abstract}
The concept of Personal Learning Environments (PLE) is, one of the concepts in the field of educational technology that has gained more attention in during the last decade. Those of us who do research on this topic assume - perhaps naively- that this is a disruptive, a fundamental concept, at the base of the emergent pedagogical approaches that characterize the techno-social age we live in, not only from the technological and instrumental perspective, but its roots in the very foundations of the educational process. Nevertheless, we know very little about the impact of this concept - and other associated topics - on other educational issues that are not necessarily linked to technology. By means of a systematized review of the available literature, we analyzed the impact of the PLE topic on three main themes of the scientific literature in education produced during the last decade: emerging pedagogical practices, self-regulated learning and professional teacher development. The analysis is focused on whether (and how) the PLE concept has impacted each of the topics, the level of importance of that impact in each one of the themes and the way in which the concept is "materialized" in them. The conclusions of this article intend to offer suggestions on the future of research and the applications in practice of a concept that, after more than ten years of its emergence in the educational field, continues to be considered as emergent.
\end{abstract}

Keywords: personal learning environment; emergent educational practices; self-regulated learning; teacher's professional development; systematized literature review.

Los Entornos Personales de Aprendizaje (en adelante PLE) representan, sin duda alguna, uno de los enfoques que mayor disrupción han causado en el ámbito de la tecnología educativa en los últimos tiempos (Castañeda, Dabbagh, y TorresKompen, 2017; Coll y Engel, 2014; Prendes y Roman, 2017).

El interés en los PLE se evidencia, además de en las numerosas publicaciones que le han dedicado un número especial a recoger artículos específicos sobre el tema (eLearning Papers en 2007 y 2013, Digital Education Review en 2010, 2011 y 2015, International Journal of Virtual and Personal Learning Environments en 2011 y 2013, Journal of Literacy and Technology en 2014, Cultura y Educación en 2014, Edutec-e en 2016, Journal of New Approaches in Educational Research en 2017, solo por nombrar algunos), en un nada despreciable número de revisiones de literatura y meta análisis alrededor del concepto. Algunas de esas revisiones son de carácter general (Buchem, Attwell, y Torres-Kompen, 2011; Chaves, 2014, 2016; Gallego y Chaves, 2014; Gallego y Gamiz, 2014; Humanante-Ramos, García-Peñalvo, y CondeGonzález, 2017; Liew y Kang, 2014; Meza Cano, Morales Ruiz, y Flores Macías, 2016; Seufert, Stanoevska-Slabeva, Mueller, y Scheffler, 2015)2016; Seufert, StanoevskaSlabeva, Mueller, \& Scheffler, 2015 y otras relacionando el tema PLE con otros temas más concretos (Dabbagh y Kitsantas, 2012; Shaikh y Khoja, 2012; Wong, 2012). 
Se trata de un interés que puede circunscribirse muy bien dentro de la última década. Aunque hay discrepancias sobre la primera referencia escrita sobre PLE (algunos autores la sitúan en 2001, otros en 2004), no fue sino hasta 2008 cuando la producción académica sobre PLE comenzó a tomar fuerza (Castañeda y Adell, 2013). Una búsqueda simple en la Web of Science arroja sólo un único resultado para las palabras clave Personal Learning Environment o PLE en 2006, en el área de Educación e Investigación en Educación; es en 2008 cuando el número de publicaciones relevantes llega a nueve; y a partir de allí comienza a aumentar gradualmente hasta los 287 , que arroja esa misma búsqueda en WOS o los 678 de SCOPUS. La década de 2008 a 2017 también incluye el congreso The PLE Conference, que durante cinco ediciones (la primera en 2010 y la última en 2014) sirvió de catalizador para la producción y divulgación científica en esta área (Hernández, 2016).

Sin embargo, a la luz de semejante desarrollo de trabajos y de interés por un tema como este, con un potencial tan aparentemente extraordinario y que afecta de forma transversal no solo a la tecnología educativa, sino a muchos aspectos subyacentes y consecuentes del proceso educativo, es relevante plantearse hasta qué punto un concepto tan bien recibido en el ámbito concreto de la tecnología educativa tiene impacto real y potencial en la educación en general, y en los grandes temas que ocupan a la investigación educativa. Surge así la gran pregunta de cuál ha sido el impacto del concepto PLE en educación -si es que ha habido alguno-. Con la intención de contestarla, se ha llevado a cabo una revisión bibliográfica sistematizada para intentar aproximar cuál es el impacto visible en la literatura científica sobre educación en general -no solo la de tecnología educativa-; y esbozar cómo se relaciona el concepto con los temas clave de la educación.

\section{ESTUDIO}

\section{Diseño metodológico}

La revisión de literatura que se presenta en este trabajo responde a una aproximación sistematizada (Grant y Booth, 2009), más propia de las ciencias sociales que las ya tradicionales revisiones sistemáticas, en tanto que más flexible metodológicamente, pero igualmente construida sobre criterios sistematizadores y de calidad (Booth, Papaioannou, y Sutton, 2012). Para realizarla, se ha seguido un procedimiento en el que se ha combinado el protocolo de ocho pasos definido por Okoli y Schabram (2010) y las fases de revisión de literatura que exponen en su trabajo McMillan y Schumaher (2009). Esa combinación ha sido utilizada con resultados muy interesantes en trabajos anteriores (Bartolomé, Castañeda, y Adell, 2018, p. 6), y en este caso han sido adaptadas con un doble proceso de muestreo (que se detalla más adelante), para responder adecuadamente a las preguntas de investigación que guían este trabajo. 
Como rango de análisis se toma como referencia la última década (2008 a 2017, ambos inclusive), entendiendo que, como se ha justificado antes, supone el periodo de desarrollo más importante de producción académica alrededor del concepto.

Las bases de datos utilizadas en la selección de los artículos son tres:

- Web of Science (en adelante WOS): consta de siete bases de datos de áreas específicas y contiene documentación científica desde 1900 en adelante. Durante más de 40 años fue la principal y única base de datos de referencia en el mundo científico y académico.

- SCOPUS: creada en 2002, es considerada junto con WOS la base de datos de referencia más utilizada en el ámbito científico y académico (Archambault, Campbell, Gingras, y Larivière, 2009; Granda-Orive et al., 2013).

- Google Scholar (Google Académico): creada en 2004, es la más reciente de las tres. Aunque su rigurosidad ha sido cuestionada por algunos autores (Jacsó, 2006). Esta base de datos cuenta con algunas ventajas importantes (Hodge y Lacasse, 2011): en primer lugar, incluye más memorias de conferencias y congresos; en segundo lugar, su índice H no está limitado a un período específico; por último, proporciona un acceso más amplio a publicaciones en el ámbito de ciencias sociales, comparado con las otras dos bases de datos.

\section{Preguntas de investigación}

- ¿Cuál ha sido el impacto cuantitativo del concepto de PLE en los temas más importantes referidos a la educación en general que ocupan la literatura científica de nuestro tiempo?

- ¿Cómo se ha materializado conceptualmente el impacto del concepto PLE en cada uno de los temas clave de la educación?

\section{Protocolo de revisión}

Selección de los temas clave

Consecuencia de las preguntas de investigación, el primer paso del proceso consistió en la delimitación de los temas más importantes referidos a la educación en general que ocupan la literatura científica de los últimos tiempos. Para ello, se realizó una búsqueda simple en WOS de todos los ítems que se clasifican en la base de datos bajo el tema "education" y se eligieron los 100 artículos más citados dentro de la propia base de datos. Se asume en este trabajo que los artículos más citados de un tema son los que más impacto posterior han tenido.

Una vez seleccionados se extrajeron las palabras clave (keywords), de las que se contabilizaron 885 , y entre las que se identificaron 593 términos únicos que 
se agruparon con términos relacionados, lo cual sirvió para realizar una primera clasificación temática en la que se incluyó la incidencia de cada uno, para valorar su importancia en la producción científica analizada.

Tras agrupar los términos, se identificaron aquellos que tenían una mayor incidencia, entendiendo que podrían entenderse como los temas de mayor impacto en la literatura especializada en educación de la última década. Así se identificaron tres grandes temas:

1. Emergent pedagogies/practices (prácticas pedagógicas emergentes) en el que se agruparon palabras clave como cooperative learning, distance education, e-leaning, expansive learning, video technology, video, virtual reality, Web 2.o, entre otros, y que acumuló un total de 139 apariciones.

2. Teacher professional development (desarrollo profesional docente), en el que se agruparon palabras clave como: learning about teaching, knowledge for teaching, teachers conceptions, teachers perceptions, entre otros, hasta llegar a un total de 51 apariciones.

3. Self-Regulated Learning (aprendizaje autorregulado), en el que se agruparon palabras clave como motivation and srl, self-regulated learning, self-regulation, self-reports, self-affirmation, self-efficacy, perceived competence entre otros, hasta acumular un total de 36 .

\section{Búsqueda temática}

Una vez identificados estos tres temas como claves, se procedió a realizar búsquedas específicas sobre cada uno de ellos tanto en la base de datos WOS como en la base de datos SCOPUS. Con el fin de perfilar y optimizar al máximo la búsqueda, cada uno de los temas clave se descompuso a su vez en sus ámbitos principales y se plantearon alternativas a cada uno de los componentes que pudieran completar la cadena de búsqueda (booleano), en un proceso que se demuestra en el siguiente ejemplo (figura 1), referido al tema de prácticas pedagógicas emergentes. 
Figura 1. Pedagogías/prácticas emergentes: delimitación del campo semántico para desarrollo de la cadena de búsqueda (booleano)

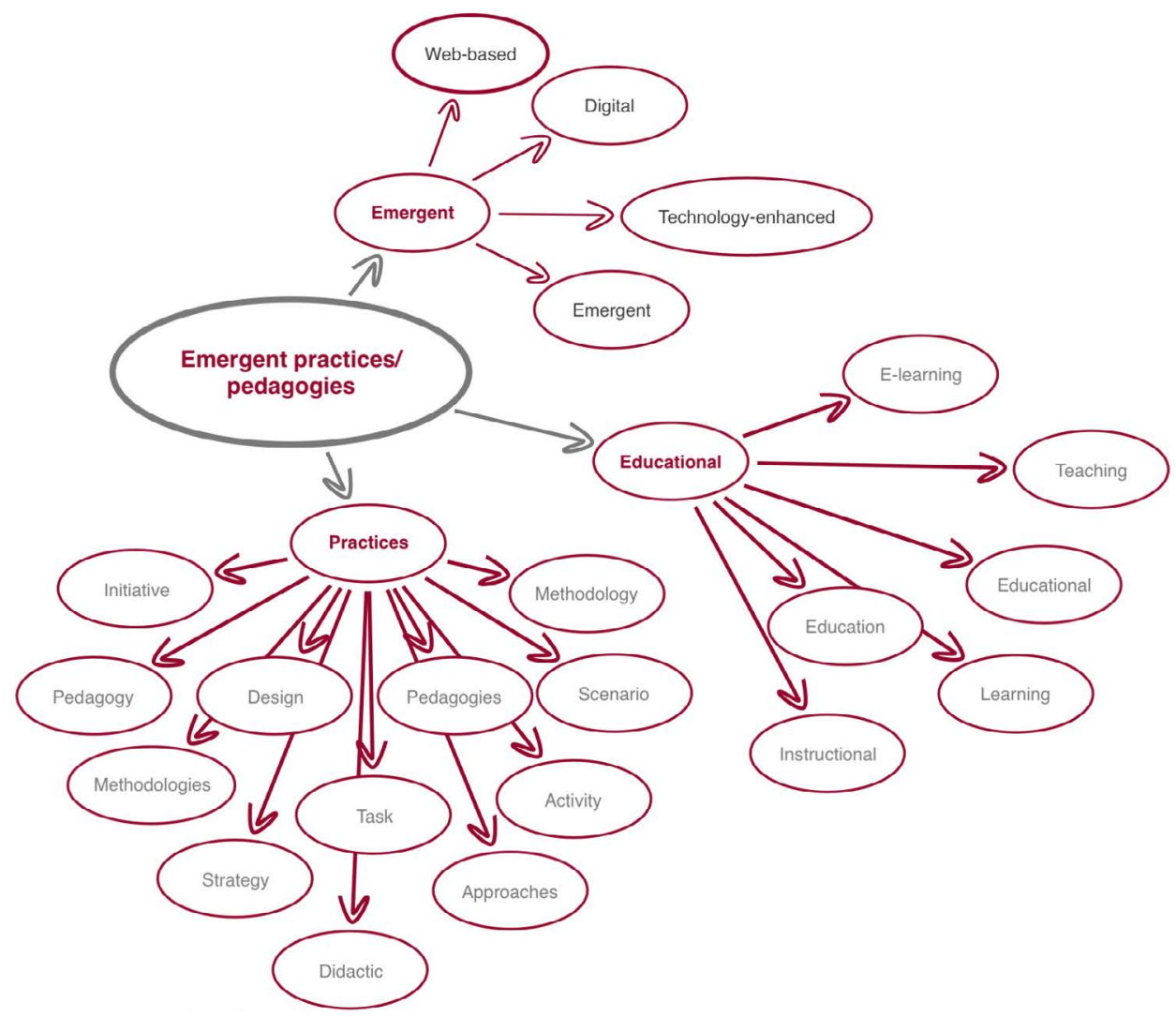

Con base en este proceso, se elaboraron las búsquedas que quedaron definidas por los siguientes booleanos, que a su vez se adaptaron a las características de búsqueda de cada una de las bases de datos. Así, por ejemplo, para la búsqueda en SCOPUS se utilizaron: 
Figura 2. Cadenas de búsqueda -booleanos- para la búsqueda en SCOPUS

\section{Pedagogías/prácticas emergentes (emergent pedagogies):}

(TITLE-ABS-KEY (emergent OR digital OR Web based OR technology enhanced) AND TITLE-ABS-KEY (Learning OR Education OR Teaching OR e-learning OR instructional OR educational) ) AND TITLE-ABS-KEY (Pedagogies OR Pedagogy OR Methodologies OR Methodology OR Didactic OR Strategy OR Design OR Scenario OR approaches OR initiative OR task OR activity) ) AND DOCTYPE ( ar ) AND PUBYEAR > 2007 AND PUBYEAR < 2018 AND (LIMIT-TO ( SUBJAREA, "SOCI "))

\section{Desarrollo profesional docente (teacher's professional development)}

(TITLE-ABS-KEY (Teacher OR Educator OR Teaching OR Trainers) AND TITLE-ABS-KEY ( learning OR education OR teaching OR instruction) AND TITLE-ABS-KEY (pedagogies OR pedagogy OR methodologies OR methodology )) AND DOCTYPE (ar) AND PUBYEAR > 2007 AND PUBYEAR < 2018 AND (LIMIT-TO (SUBJAREA, "SOCI"))

\section{Aprendizaje autorregulado (selfregulated learning):}

(TITLE-ABS-KEY ( selfregulated OR self-regulated OR self-determined OR self-motivated OR self-regulation OR selfregulation OR auto-regulation OR auto-regulated OR autoregulation OR autoregulated) AND TITLEABS-KEY (learning OR education OR training OR learners OR students)) AND DOCTYPE (ar) AND PUBYEAR $>2007$ AND PUBYEAR < 2018 AND ( LIMIT-TO (SUBJAREA, "SOCI" ))

Como se deduce de las cadenas de búsqueda, la revisión se circunscribió solo a artículos de revista, del ámbito de las ciencias sociales en el caso de SCOPUS, y a las categorías explícitamente nombradas como la de educación, en el caso de WOS.

\section{Primer muestreo}

Tras esta primera exploración en las bases de datos especializadas, se recogieron dos tipos de muestras temáticas que se estudiaron de manera paralela:

- Muestra de los artículos de mayor impacto en cada temática: incluye los 200 artículos más citados en cada una de las bases de datos en cada uno de los temas.

- Muestra de incidencia de PLE en la temática: todos los artículos referidos a "Personal Learning Environment" en cada base de datos, diferenciados por tema. 


\section{Segundo muestreo}

Cada uno de los tipos de muestras se trató de forma diferenciada; así:

- De la muestra de los artículos de mayor impacto en cada temática, se extrajeron datos genéricos y se analizó cuántos artículos estaban relacionados con el tema Personal Learning Environment a partir de los datos principales con que están indizados en las bases de datos (palabras clave, resumen, referencias y título).

- En el caso de las muestras de incidencia de PLE en la temática se hizo un trabajo más minucioso que consistió en: unificación y descarte de los repetidos, análisis del impacto usando como fuente de citación Google Académico, y clasificación de los artículos en cada una de las bases con relación a ese impacto. Tras realizar un análisis del índice $\mathrm{H}$ de la muestra, se eligieron para el análisis más profundo los 20 artículos con mayor impacto en citas, y con ellos se procedió a realizar un análisis de contenido que respondiese a las preguntas de investigación.

\section{Protocolo de análisis}

A partir de la selección de los artículos que conforman las tres colecciones temáticas, se procedió a su lectura y análisis, y se hizo una recogida de datos mixtos (cualitativos y cuantitativos) a partir de un instrumento, una ficha de observación, que se creó $a d$ hoc. La ficha se validó mediante su implementación piloto por parte de los investigadores, para asegurar su univocidad, además de para unificar los procedimientos de análisis y recogida de evidencias cualitativas.

\section{DATOS}

\section{Datos del primer muestreo}

\section{Muestra general}

La primera parte de la búsqueda evidenció la realidad de las referencias a los PLE en los tres temas identificados como centrales en este análisis, así: 
Tabla 1. Muestra general de cada temática en las bases de datos WOS y SCOPUS (20072017). Datos generales

\begin{tabular}{|c|c|c|c|c|c|}
\hline Tema & $\begin{array}{l}\text { Base de } \\
\text { Datos }\end{array}$ & $\begin{array}{c}\text { Total } \\
\text { artículos }\end{array}$ & \multicolumn{2}{|c|}{ Referidos a PLE } & $\begin{array}{c}\text { Artículos sobre PLE entre } \\
\text { los } 200 \text { más citados de la } \\
\text { base de datos }\end{array}$ \\
\hline \multirow{3}{*}{ Emergent Pedagogies } & SCOPUS & 9441 & 155 & $1,64 \%$ & 4 \\
\hline & WOS & 6193 & 18 & $0,29 \%$ & o \\
\hline & & Total & \multicolumn{2}{|c|}{$\begin{array}{c}164 \text { sin } \\
\text { repeticiones }\end{array}$} & 4 \\
\hline \multirow{3}{*}{$\begin{array}{l}\text { Teachers Professional } \\
\text { Development }\end{array}$} & SCOPUS & 86448 & 285 & $0,32 \%$ & o \\
\hline & WOS & 115172 & 26 & $0,02 \%$ & o \\
\hline & & Total & \multicolumn{2}{|c|}{$\begin{array}{c}303 \sin \\
\text { repeticiones }\end{array}$} & $\mathbf{o}$ \\
\hline \multirow{3}{*}{$\begin{array}{l}\text { Self-regulated } \\
\text { learning }\end{array}$} & SCOPUS & 2960 & 59 & $2 \%$ & 4 \\
\hline & WOS & 2322 & 9 & $0,38 \%$ & 2 \\
\hline & & Total & \multicolumn{2}{|c|}{$\begin{array}{c}65 \mathrm{sin} \\
\text { repeticiones }\end{array}$} & 4 \\
\hline
\end{tabular}

Como se muestra en la tabla 1, el impacto del concepto PLE en los artículos que se han indexado en cada una de las bases de datos estudiadas, relacionados con cada uno de los temas identificados como cruciales en la literatura científica sobre educación, es ciertamente desigual.

En el caso de los artículos indexados como relacionados con el desarrollo profesional docente (Teacher's Profesional Development, en adelante TPD), aunque hemos encontrado hasta 303 artículos que están relacionados con PLE en ambas bases de datos, ninguno se sitúa dentro de los 200 más citados de sus respectivas bases de datos.

Por su parte, en el caso de los artículos relativos a aprendizaje autorregulado (Self-Regulated Learning, en adelante SRL) y a los artículos relacionados con las pedagogías o prácticas educativas emergentes (Emergent Pedagogies, en adelante EP), la aparición del concepto PLE en los artículos indexados es bastante similar.

En ambos casos (EP y SRL) llama la atención que de los artículos más influyentes de la temática en la última década hay cuatro en cada colección que incluyen el concepto PLE; y que entre los cuatro acumulan más de 1000 citas en Google Académico ${ }^{1}$ en cada caso (1294 en el caso de los 4 referidos a EP y 2039 en el de SRL). 


\section{Muestra de incidencia del tema PLE en cada temática}

Realizada una segunda búsqueda dentro de cada base de datos para buscar aquellos artículos relacionados con PLE en cada una de las temáticas, y una vez descartadas las repeticiones, obtuvimos una muestra de:

- 164 artículos relacionados con EP en ambas bases de datos, 132 de los cuales (el 8o,48\%) tenían citas en Google Académico, y con un índice $\mathrm{H}^{2}$ (en adelante $\mathrm{iH}$ ) de 19, según los datos de esa misma base de datos.

- En el caso del TPD se localizaron 303, 96 de ellos (un 31,68\%) con citas y con un iH de 31.

- Y 65 artículos en el caso de SRL, 49 con citas (75,38\%) y un iH de 27.

\section{Datos del segundo muestreo}

\section{Datos generales}

De las colecciones fruto del segundo muestreo, es decir, de aquellas que recogen los 20 artículos más citados relacionados con cada temática (aunque por razones de conveniencia se incluyen los 21 primeros en el caso de SLR y de TPD), podemos hacer un primer análisis cuyos datos generales se muestran en la tabla 2:

Tabla 2. Datos generales, colecciones fruto del segundo muestreo

\begin{tabular}{|l|c|c|c|c|c|}
\hline Colección & $\mathrm{N}$ & $\begin{array}{c}\text { Índice H de la } \\
\text { colección }\end{array}$ & $\begin{array}{c}\text { Citas acumuladas } \\
\text { en G. Académico }\end{array}$ & $\begin{array}{c}\text { Artículos } \\
\text { empíricos }\end{array}$ & $\begin{array}{c}\text { Si es empírico des una } \\
\text { experiencia didáctica? }\end{array}$ \\
\hline EP & 20 & 19 & 2514 & 10 & 7 \\
\hline TPD & 21 & 31 & 1652 & 11 & 6 \\
\hline SRL & 21 & 27 & 2551 & 13 & 11 \\
\hline
\end{tabular}

Como se puede ver en la tabla 2, de la veintena recogida en cada colección ${ }^{3}$, en torno a la mitad de ellos son artículos que toman como base una investigación empírica; y no en todos los casos la investigación incluye una experiencia didáctica, es decir, involucra un proceso de enseñanza y/o aprendizaje.

En lo que se refiere a los PLE, como podemos observar en los datos de la tabla 3 , entre un $60 \%$ y un 70\% de los artículos de las colecciones incluyen explícitamente el término Personal Learning Environment o PLE en el texto, aunque la mitad de los referidos a SRL no explicitan una definición de lo que es PLE, dato que sube hasta un 70-75\% en el caso de las colecciones sobre EP y TPD.

En la mayoría de los veinte artículos referidos a EP y SRL se usa un enfoque de PLE a medio camino entre lo tecnológico y lo pedagógico en el ámbito de la 
complementariedad. No obstante, en el caso de la colección relacionada con el TPD los artículos se distribuyen casi por igual en las tres opciones.

Tabla 3. Aparición del concepto PLE en cada una de las colecciones

\begin{tabular}{|c|c|c|c|c|}
\hline Colección & $\begin{array}{c}\text { Aparece explícitamente el } \\
\text { término Personal Learning } \\
\text { Environment o PLE }\end{array}$ & $\begin{array}{c}\text { Enfoque } \\
\text { Pedagógico } \\
\text { de PLE }\end{array}$ & $\begin{array}{c}\text { Enfoque } \\
\text { tecnológico } \\
\text { de PLE }\end{array}$ & $\begin{array}{c}\text { Enfoque } \\
\text { intermedio } \\
\text { del PLE }\end{array}$ \\
\hline EP & 14 & 2 & 1 & 17 \\
\hline TPD & 12 & 7 & 8 & 6 \\
\hline SRL & 12 & 6 & 2 & 13 \\
\hline
\end{tabular}

Además, en la mayoría de trabajos se recogen las aportaciones de otros autores (aunque la mayoría de ellos no forman parte de ninguna de las colecciones incluidas en el muestreo) para justificar y conceptualizar PLE. De entre ellos es imprescindible destacar la influencia de los trabajos de Attwell (Attwell, 2007; Attwell, Cook, y Ravenscroft, 2009), con una especial mención al trabajo de 2007 que aparece citado en más de diez artículos de los incluidos en las diferentes colecciones, los trabajos de Downes (2005, 2007), los de Taraghi, Ebner y diferentes equipos (Ebner y Taraghi, 2010; Taraghi, Ebner, y Schaffert, 2009; Taraghi, Ebner, Till, y Mühlburger, 2009), los de Adell y Castañeda (Adell y Castañeda, 2010; Castañeda y Adell, 2013), los de Casquero y colaboradores (Casquero, 2013; Casquero, Portillo, Ovelar, Romo, y Benito, 2008).

Datos de la colección PLE en pedagogías emergentes (EP):

De los 20 artículos analizados, cuatro se consideran como de mucha influencia en relación con la temática EP (Drexler, 2010; Fini, 2009; Kop, Fournier, y Mak, 2011; Wong, 2012).

Todos los artículos considerados de esta colección se refieren a propuestas de modelos o marcos (frameworks) para el diseño del proceso de aprendizaje; algunos, orientados a la construcción de PLE y otros al uso de PLE como herramientas en el marco de un modelo específico.

Estos artículos también tienen en común que, aunque incluyen una perspectiva complementaria (tecnológica-pedagógica), predomina un enfoque más pedagógico, y la infraestructura utilizada para crear o acceder a los PLE (la visión del PLE como un objeto) toma un segundo plano respecto al modelo pedagógico que hace uso del PLE (la visión del PLE como un concepto).

Las soluciones tecnológicas, como era de esperarse, van cambiando junto con el escenario tecnológico que corresponde a cada artículo. Barrett y Garret (2009) asocian los PLE a e-portfolios; Williams (2008) menciona social bookmarking 
y mundos virtuales (Second Life) como parte de las aplicaciones comúnmente utilizadas por los estudiantes; Drexler (2010) visualiza la construcción de PLE a través de blogs y Google Reader entre otras aplicaciones; Chatti y su equipo (2010; 2010)it has been argued that technology-enhanced learning (TEL proponen PLE basados en mashups; y Wong (2012) los asocia a mobile learning.

Datos de la colección PLE en desarrollo profesional docente (TPD):

De los 21 artículos analizados, en el texto de once de ellos aparece literalmente el término Personal Learning Environment, aunque solo seis explicitan una definición del mismo, casi siempre apoyado en trabajos de otros autores, pero remarcando de forma especial la naturaleza emancipadora de los PLE como herramienta de autoorganización para el aprendiz.

Entendemos que ocho artículos de la colección asumen una definición más tecnológica de los PLE, seis de los artículos evidencian una visión más pedagógica del concepto de PLE, y seis artículos incluyen rasgos tecnológicos y pedagógicos en su visión de PLE, casi siempre de forma complementaria, aunque no siempre consciente.

En todo caso, resulta tremendamente llamativo que, de los 21 artículos analizados, todos clasificados por ambas bases de datos (SCOPUS y WOS) como relacionados con TPD, solo hay tres que aborden el desarrollo profesional docente de forma directa (De Smet, Bourgonjon, De Wever, Schellens, y Valcke, 2012; Kellogg, Booth, y Oliver, 2014; Oakley, Pegrum, y Johnston, 2014).

En esta colección, la inmensa mayoría de los artículos estudiados (14) entiende que los PLE vienen a modificar "lo que un profesor debe saber" para ser mejor docente en el contexto que se mueve, sea este universitario como en Valjataga y Laanpere (2010), de primaria como en Lai, Hwang y Lei (2012), o genérico, como el caso de Rodríguez-Triana, Martínez-Monés, Asensio-Pérez y Dimitriadis (2015); en casi todos ellos se entiende que la formación del profesorado en el uso de las TIC debe incluir el PLE bien como metodología, bien como entorno tecnológico, o bien como enfoque de cambio cualitativo y metodológico de entendimiento del rol profesor-alumno (González-Martínez, Bote-Lorenzo, Gómez-Sánchez, y CanoParra, 2015; Hrastinski y Aghaee, 2012): siempre como un contenido a incluirse en el TPD, aunque no se especifique cómo.

Finalmente, destacan dos artículos que sí relacionan el PLE con cambios sustanciales en el TPD: el de Oakley et al. (2014), donde los PLE se enuncian como enfoque y herramienta clave en la formación del profesorado; y el de Jovanovic et al. (2009), donde los PLE aparecen explícitamente como nuevo paradigma de organización del aprendizaje no solo de los estudiantes, sino de los profesores; y aunque solo se habla de la tecnología que lo sustenta (Semantic Web Tools), se hace mención explícita de estas herramientas como herramientas para hacer TPD desde la perspectiva del desarrollo de los PLE de los profesores. Ambos artículos ponen 
al profesor como aprendiz en el centro nuclear de su formación y entienden que los PLE son su propia aproximación al aprendizaje.

\section{Datos de la colección PLE en aprendizaje autorregulado (SRL)}

Los cuatro artículos más citados de esta temática (Dabbagh y Kitsantas, 2012; Johnson y Liber, 2008; McLoughlin y Lee, 2010; Meyer, Abrami, Wade, Aslan, y Deault, 2010)(b se encuentran no solo entre los 200 más citados sobre SRL en SCOPUS y WOS, sino entre los 50 más citados en total. Además, estos artículos (Dabbagh y Kitsantas, 2012, McLoughlin y Lee, 2010 y, en menor medida, Johnson y Liber 2008) fundamentan teóricamente otros artículos de la misma colección centrados en el concepto PLE y, como hemos visto, con gran influencia en el campo.

De los 21 artículos de la colección, seis abordan el tema del aprendizaje autorregulado desde entornos digitales (Lai y Hwang, 2016; Shen, Lee, y Tsai, 2011; Tsai, 2010), argumentando la personalización del proceso de aprendizaje -y el PLE - tanto por las posibilidades desde el punto de vista tecnológico como del diseño pedagógico. Asumen estos textos que se planifica con diversidad de metodologías y estrategias didácticas, y que pueden incluir en algunos casos las herramientas propias de la web social (Cho y Cho, 2013; Kitsantas, 2013; Lin, Huang, y Chuang, 2015). Mención especial merecen los tres artículos centrados exclusivamente en portafolios electrónicos (Abrami, Venkatesh, Meyer, y Wade, 2013; Chang, Tseng, Liang, y Liao, 2013; Meyer et al., 2010), que emergen como estrategia didáctica genuina de fomento de las habilidades cognitivas superiores desde entornos digitales.

En los seis artículos que explícitamente desarrollan el concepto de PLE y que podemos analizar desde una perspectiva más pedagógica, los entornos digitales se generan a partir de la inclusión de los social media que se conciben para la propiedad del aprendiz y el trabajo de habilidades cognitivas relacionadas con el aprendizaje autorregulado. Así, en estos trabajos el concepto de PLE suele incluir la referencia a los social media y las posibilidades para la creación y compartición de conocimiento que en definitiva se relaciona con el control del aprendizaje por parte del estudiante (Rahimi, Van Den Berg, y Veen, 2015).

Sólo el artículo de Taraghi (2012) aborda el PLE desde un punto de vista meramente tecnológico, con una propuesta de entorno compatible con dispositivos móviles cuyas posibilidades, según el autor, podrían aumentar y fomentar las habilidades para el aprendizaje autorregulado. 


\section{CONCLUSIONES}

Tratando en primer lugar de responder a nuestras preguntas de investigación, cabe decir que efectivamente el ámbito de estudio de los PLE ha impactado de manera importante en la literatura sobre educación en general. Este hecho se refleja principalmente en que dos de los tres campos de mayor relevancia en las publicaciones de la última década, aquellos estudios relacionados con las prácticas pedagógicas emergentes (EP) y con el aprendizaje autorregulado (SRL) contemplan como enfoque el PLE en los artículos de mayor impacto. En la otra cara de la moneda, también hemos de decir que no es el caso del desarrollo profesional docente, donde la influencia de los PLE no se ha dejado notar, al menos entre los artículos de mayor impacto posterior.

En lo que respecta a las EP, el concepto PLE ha permitido evolucionar hacia la propuesta de modelos de aprendizaje que intentan romper con estructuras tradicionales; que se enfocan en el aprendiz (hasta el punto de ubicarlo en el centro del proceso de aprendizaje) y le dan más independencia. En contraste, la institución y los profesores adoptan un papel de facilitadores y guías del proceso.

Como bien nos demuestran los datos, si bien hay parte de la literatura sobre TPD que es consciente de la existencia de los PLE, casi toda ella entiende que son parte de lo que el profesor debería dominar, bien como herramienta de enseñanza, como entorno de sus estudiantes para aprender, o como enfoque metodológico; pero solo una parte muy reducida de esa literatura entiende que los PLE son además otra forma de enfocar el propio TPD. Esto en parte conecta con algunas de las percepciones evidentes de los docentes respecto del TPD, que parece siempre ocuparse de cuál es el contenido que le concierne pero que no siempre se ocupa de integrar lo que sabemos sobre pedagogía y sobre formación en la propia formación del profesorado.

La visión global de la temática de la colección sobre SRL nos indica que los PLE, entendidos desde el punto de vista pedagógico han incidido claramente en el marco conceptual del aprendizaje autorregulado, si bien esta afirmación permite matices. Por una parte, los entornos digitales se convierten en instrumentos que se conciben para el control del aprendizaje por parte del aprendiz, gracias a las herramientas que muchas veces se basan en servicios de la Web 2.o.; y para el trabajo de habilidades cognitivas relacionadas con el aprendizaje autorregulado. Por lo tanto, a pesar de esta voluntad de diseño pedagógico preocupado por la autonomía del estudiante en el desarrollo de su proceso, la visión del entorno nos puede llevar a considerar un cierto rol instrumental del PLE en algunos trabajos.

Por otra parte, y superando este marco psicológico del SRL y su consiguiente rol instrumental de la tecnología, las posibilidades de los PLE para borrar los límites entre aprendizaje formal e informal nos llevan a modelos centrados en la agencia del aprendiz, el modelo aprendizaje autónomo, el independiente, o la heutagogía o aprendizaje autodirigido (self-directed learning). Estas valoraciones generales nos permiten llegar a la conclusión de que el concepto PLE ha aportado al modelo del SRL, 
en primer lugar, una estrategia que permite el diseño enriquecido del aprendizaje; y en segundo lugar, cabe destacar que ha permitido una visión transformadora del entorno del aprendizaje en el que el aprendiz ejerce un rol activo que le permite asumir la agencia de sus propios procesos a lo largo y ancho de la vida.

Cuando analizamos de manera general la producción científica relacionada con PLE en cada uno de estos tres ámbitos de interés que hemos identificado como claves en la publicación educativa especializada de los últimos diez años, de manera general vemos que muchos de los artículos que hablan de PLE no lo definen explícitamente. Es posible que el concepto haya alcanzado una madurez suficiente como para incorporarse al lenguaje no sólo de académicos e investigadores, sino también de profesores y aprendices.

En ese análisis general vemos reflejada también la ambigüedad del concepto PLE, o lo que es lo mismo: que sigue habiendo una literatura que continúa entendiéndolo como una realidad tecnológica y otra como una realidad pedagógica; sin embargo, la inmensa mayoría de los artículos analizados nos muestran que el análisis del PLE como una realidad mixta es la opción más relevante, abriéndose además a la posibilidad de afrontar el análisis de esa realidad desde perspectivas fenomenológicas o incluso post-fenomenológicas, como las propias de las perspectivas socio-materiales.

Cabe destacar que poco más de la mitad de la literatura más citada sobre los tres temas que nos ocupan está basada en estudios empíricos y que de estos solo un $39 \%$ son experiencias educativas, aunque la mayoría de ellas con grupos reducidos o análisis limitados (con métricas referidas a la satisfacción, la mucha o poca interacción o a la motivación). Teniendo en cuenta que se trata de los artículos más citados del tema - y por lo mismo más influyentes- entendemos que debería hacerse una apuesta más importante por artículos que se planteen soportes teóricos mucho más profundos, pero además con una perspectiva de análisis mucho más compleja que nos permitan realmente avanzar o proponer nuevos caminos para analizar asuntos educativos que, sin lugar a dudas, son de gran importancia. Dicho de otra forma, seguimos echando en falta estudios que desarrollen el potencial analítico del PLE y que busquen entender la realidad que da cabida a los PLE desde propuestas metodológicas ambiciosas.

Es evidente que el tema PLE sigue siendo parte del cuerpo de las tecnologías/ pedagogías emergentes (Veletsianos, 2016) y que sigue en un estado de potencial aún sin desarrollar completamente. Ahora bien, si algo evidencia la literatura es que los PLE han impactado de forma frontal y muy importante en los temas más importantes de la investigación educativa. Dos de los ámbitos más relevantes en la publicación educativa incluyen en sus artículos más impactantes la perspectiva PLE y eso nos da una buena medida de su importancia, máxime teniendo en cuenta que ha tenido un tiempo de desarrollo relativamente corto. No obstante, su potencial está aún por desarrollarse, su ausencia en los artículos de mayor impacto en un tema como el TPD supone un reto evidente para quienes nos seguimos dedicando a este tema y un aliciente para proponer perspectivas más holísticas, que aborden no 
solo el PLE dentro de la tecnología educativa al uso, sino también dentro de todo el andamiaje pedagógico y educativo.

Es tiempo de continuar.

\section{NOTAS}

1. Datos de citación recogidos en Google Académico a fecha 25 de febrero de 2018.

2. El índice $h$ es el balance entre el número de publicaciones y las citas a estas.

3. La lista completa de los artículos que componen este segundo muestreo está disponible en https://bit.ly/2IbcbZK

\section{REFERENCIAS}

Abrami, P. C., Venkatesh, V., Meyer, E. J., y Wade, C. A. (2013). Using electronic portfolios to foster literacy and selfregulated learning skills in elementary students. Journal of Educational Psychology, 105(4), 1188-1209. doi: https://doi.org/10.1037/a0032448

Adell, J., y Castañeda, L. (2010). Los Entornos Personales de Aprendizaje (PLEs): una nueva manera de entender el aprendizaje. En R. Roig-Vila y M. Fiorucci (Eds.), Claves para la investigación en innovación y calidad educativa. La integración de las Tecnologías de la Información y la comunicación y la Interculturalidad en las aulas. Alcoi Roma: Marfil y Roma Tre.

Attwell, G. (2007). Personal Learning Environments - the future of eLearning? ELearning Papers, 2, 8.

Attwell, G., Cook, J., y Ravenscroft, A. (2009). Appropriating Technologies for Contextual Knowledge: Mobile Personal Learning Environments. En Best Practices for the Knowledge Society. Knowledge, Learning, Development and Technology for All (15-25). Springer, Berlin, Heidelberg. doi: https://doi. org/10.1007/978-3-642-04757-2 2

Barrett, H. C., y Garrett, N. (2009). Online personal learning environments: Structuring electronic portfolios for lifelong and life-wide learning. On the Horizon, 17(2), 142-152. doi: https://doi. org/10.1108/10748120910965511

Bartolomé, A., Castañeda, L., y Adell, J. (2018). Personalisation in educational technology: the absence of underlying pedagogies. International Journal of Educational Technology in Higher Education, 15(1). doi: https://doi. org/10.1186/s41239-018-0095-0

Booth, A., Papaioannou, D., y Sutton, A. (2012). Systematic Approaches to a Successful Literature Review (Edición: 1). Los Angeles; Thousand Oaks, Calif: SAGE Publications Ltd.

Casquero, O. (2013). Una perspectiva tecnológica. En L. Castañeda y J. Adell (Eds.), Entornos personales de aprendizaje: Claves para el ecosistema educativo en red. (71-84). Alicante: Marfil.

Casquero, O., Portillo, J., Ovelar, R., Romo, J., y Benito, M. (2008). iGoogle and gadgets as a platform for integrating institutional and external services. En Proceedigns of the Workshop on MashUp Personal Learning Environments (MUPPLE'O8) (pp. 37-41). Maastrich, The Netherlands.

Castañeda, L., y Adell, J. (Eds.). (2013). Entornos personales de aprendizaje: 
Claves para el ecosistema educativo en red. Alicante: Marfil.

Castañeda, L., Dabbagh, N., y TorresKompen, R. (2017). Personal Learning Environments: Research-Based Practices, Frameworks and Challenges. Journal of New Approaches in Educational Research, 6(1), 1-2. doi: https://doi. org/10.7821/naer.2017.1.229

Chang, C.-C., Tseng, K.-H., Liang, C., y Liao, Y.-M. (2013). Constructing and evaluating online goal-setting mechanisms in webbased portfolio assessment system for facilitating self-regulated learning. Computers and Education, 69, 237249. doi: https://doi.org/10.1016/j. compedu.2013.07.016

Chatti, M. A., Agustiawan, M. R., Jarke, M., y Specht, M. (2010). Toward a personal learning environment framework. International Journal of Virtual and Personal Learning Environments, 1(4), 66-85. doi: https://doi.org/10.4018/ jvple.2010100105

Chatti, M. A., Jarke, M., y Specht, M. (2010). The $3 \mathrm{P}$ Learning Model. Educational Technology y Society, 13(4), 74-85.

Cho, K., y Cho, M.-H. (2013). Training of self-regulated learning skills on a social network system. Social Psychology of Education, 16(4), 617-634. doi: https:// doi.org/10.1007/s11218-013-9229-3

Coll, C., y Engel, A. (2014). Introduction: Personal Learning Environments in the context of formal education / Introducción: los Entornos Personales de Aprendizaje en contextos de educación formal. Cultura y Educación, 26(4), 617630. doi: https://doi.org/10.1080/113564 05.2014.985947

Dabbagh, N., y Kitsantas, A. (2012). Personal Learning Environments, social media, and self-regulated learning: A natural formula for connecting formal and informal learning. The Internet and Higher Education, 15(1), 3-8. doi: https://doi. org/10.1016/j.iheduc.2011.06.002
De Smet, C., Bourgonjon, J., De Wever, B., Schellens, T., y Valcke, M. (2012). Researching instructional use and the technology acceptation of learning management systems by secondary school teachers. Computers y Education, 58(2), 688-696. doi: https://doi.org/10.1016/j. compedu.2011.09.013

Downes, S. (2005, octubre). E-learning 2.o. eLearn Magazine, an ACM Publication. Recuperado de https://elearnmag.acm. org/featured.cfm?aid $=1104968$

Downes, S. (2007, junio). Open Educational Resources and The Personal Learning Environment. Tecnología presentado en International Conference on OpenCourseWare, Taipei. Recuperado de https://es.slideshare.net/Downes/openeducational-resources-and-the-personallearning-environment

Drexler, W. (2010). The networked student model for construction of personal learning environments: Balancing teacher control and student autonomy. Australasian Journal of Educational Technology, 26(3). doi: https://doi. org/10.14742/ajet.1081

Ebner, M., y Taraghi, B. (2010). Personal Learning Environment for Higher Education - A First Prototype. En Proceedings of th World Conference on Educational Multimedia, Hypermedia and Telecommunications (pp. 1158-1166). Toronto.

Fini, A. (2009). The technological dimension of a massive open online course: The case of the CCKo8 course tools. International Review of Research in Open and Distance Learning, 10 (5 SPL.ISS.). Recuperado de https://www.scopus.com/inward/record. uri?eid=2-s2.0-79956312929ypartnerID $=40 \mathrm{ymd}_{5}=627 \mathrm{aagd} 99916 \mathrm{odc}_{4}$ acdefd $3 \mathrm{~b}_{7}$ 103b164

González-Martínez, J. A., Bote-Lorenzo, M. L., Gómez-Sánchez, E., y Cano-Parra, R. (2015). Cloud computing and education: A state-of-the-art survey. Computers and 
Education, 8o, 132-151. doi: https://doi. org/10.1016/j.compedu.2014.08.017

Grant, M. J., y Booth, A. (2009). A typology of reviews: an analysis of 14 review types and associated methodologies. Health Information and Libraries Journal, 26(2), 91-108. doi: https://doi. org/10.1111/j.1471-1842.2009.00848.x

Hernández, M. A. (2016). Gestión del conocimiento, actividad científica y entornos personales de apredizaje (PLEs): una bibliometría de la PLE conference. Edutec. Revista Electrónica de Tecnología Educativa, o(55), 329.

Hrastinski, S., y Aghaee, N. M. (2012). How are campus students using social media to support their studies? An explorative interview study. Education and Information Technologies, 17(4), 451-464. doi: https://doi.org/10.1007/ s10639-011-9169-5

Johnson, M., y Liber, O. (2008). The Personal Learning Environment and the human condition: from theory to teaching practice. Interactive Learning Environments, 16(1), 3-15. doi: https:// doi.org/10.1080/10494820701772652

Jovanović, J., Gašević, D., Torniai, C., Bateman, S., y Hatala, M. (2009). The social semantic web in intelligent learning environments: State of the art and future challenges. Interactive Learning Environments, 17(4), 273-309. doi: https://doi. org/10.1080/10494820903195140

Kellogg, S., Booth, S., y Oliver, K. (2014). A social network perspective on peer supported learning in MOOCs for educators. International Review of Research in Open and Distance Learning, 15(5), 263-289.

Kitsantas, A. (2013). Fostering college students' selfregulated learning with learning technologies. Hellenic Journal of Psychology, 10(3), 235-252.

Kop, R., Fournier, H., y Mak, J. S. F. (2011). A Pedagogy of Abundance or a Pedagogy to Support Human Beings? Participant Support on Massive Open Online Courses. International Review of Research in Open and Distance Learning, 12(7), 7493. doi: https://doi.org/10.19173/irrodl. v12i7.1041

Lai, C., Wang, Q., y Lei, J. (2012). What factors predict undergraduate students' use of technology for learning? A case from Hong Kong. Computers and Education, 59(2), 569-579. doi: https:// doi.org/10.1016/j.compedu.2012.03.006

Lai, C.-L., y Hwang, G.-J. (2016). A selfregulated flipped classroom approach to improving students' learning performance in a mathematics course. Computers and Education, 100, 126-140. doi: https://doi. org/10.1016/j.compedu.2016.05.006

Lin, J.-W., Huang, H.-H., y Chuang, Y.-S. (2015). The impacts of network centrality and self-regulation on an e-learning environment with the support of social network awareness. British Journal of Educational Technology, 46(1), 32-44. doi: https://doi.org/10.1111/bjet.12120

McLoughlin, C., y Lee, M. J. W. (2010). Personalised and self-regulated learning in the Web 2.0 era: International exemplars of innovative pedagogy using social software. Australasian Journal of Educational Technology, 26(1), 28-43.

McMillan, J. H., y Schumacher, S. (2009). Research in Education: Evidence-Based Inquiry (7 edition). Boston: Pearson.

Meyer, E., Abrami, P. C., Wade, C. A., Aslan, O., y Deault, L. (2010). Improving literacy and metacognition with electronic portfolios: Teaching and learning with ePEARL. Computers and Education, 55(1), 84-91. doi: https://doi. org/10.1016/j.compedu.2009.12.005

Oakley, G., Pegrum, M., y Johnston, S. (2014). Introducing e-portfolios to pre-service teachers as tools for reflection and growth: lessons learnt. Asia-Pacific Journal of Teacher 
Education, 42(1), 36-50. doi: https://doi. org/10.1080/1359866X.2013.854860

Okoli, C., y Schabram, K. (2010). A Guide to Conducting a Systematic Literature Review of Information Systems Research. SSRN Electronic Journal. doi: https:// doi.org/10.2139/ssrn.1954824

Prendes, M. P., y Roman, M. (2017). Entornos personales de aprendizaje: Una visión actual de cómo aprender con tecnologías ( $1^{\mathrm{a}}$ ed.). Barcelona: Editorial Octaedro, S.L.

Rahimi, E., Van Den Berg, J., y Veen, W. (2015). Facilitating student-driven constructing of learning environments using Web 2.0 personal learning environments. Computers and Education, 81, 235-246. doi: https://doi. org/10.1016/j.compedu.2014.10.012

Rodríguez-Triana, M. J., Martínez-Monés, A., Asensio-Pérez, J. I., y Dimitriadis, Y. (2015). Scripting and monitoring meet each other: Aligning learning analytics and learning design to support teachers in orchestrating CSCL situations. British Journal of Educational Technology, 46(2), 330-343. doi: https://doi. org/10.1111/bjet.12198

Shen, P.-D., Lee, T.-H., y Tsai, C.-W. (2011). Applying blended learning with web-mediated self regulated learning to enhance vocational students' computing skills and attention to learn. Interactive Learning Environments, 19(2), 193-209. doi: https://doi. org/10.1080/10494820902808958

Taraghi, B. (2012). Ubiquitous Personal Learning Environment (UPLE). International Journal of Emerging Technologies in Learning (IJET), $7(\mathrm{~S} 2)$, 7-14.
Taraghi, B., Ebner, M., y Schaffert, S. (2009). Personal Learning Environments for Higher Education: A Mashup Based Widget Concept. En Proceedings of the Second International Workshop on Mashup Personal Learning Environments (MUPPLEO9) (p. 8). Nice, France.

Taraghi, B., Ebner, M., Till, G., y Mühlburger, H. (2009). Personal Learning Environment - a Conceptual Study. Presentado en International Conference on Interactive Computer Aided Learning (ICL 2009), Villach, Australia.

Tsai, C.-W. (2010). The effects of feedback in the implementation of web-mediated selfregulated learning. Cyberpsychology, Behavior, and Social Networking, 13(2), 153-158. doi: https://doi.org/10.1089/ cyber.2009.0267

Valjataga, T., y Laanpere, M. (2010). Learner control and personal learning environment: a challenge for instructional design. Interactive Learning Environments, 18(3), 277-291. doi: https://doi.org/10.1080/10494820.2010 .500546

Veletsianos, G. (2016). Emergence and innovation in digital learning: Foundations and applications. Athabasca University Press.

Williams, P. (2008). Leading schools in the digital age: a clash of cultures. School Leadership y Management, 28(3), 213-228. doi: https://doi. org/10.1080/13632430802145779

Wong, L.H. (2012). A learner-centric view of mobile seamless learning. British Journal of Educational Technology, 43(1), E19-E23. doi: https://doi.org/10.1111/ j.1467-8535.2011.01245.X 


\section{PERFIL ACADÉMICO Y PROFESIONAL DE LOS AUTORES}

Linda Castañeda. Doctora en Tecnología Educativa por la Universitat de les Illes Balears. Profesora del Departamento de Didáctica y Organización Escolar de la Universidad de Murcia y miembro del Grupo de Investigación de Tecnología Educativa (GITE) de la misma universidad. Su investigación se interesa especialmente por la implementación de pedagogías emergentes en contextos formales y no formales, Entornos personales de Aprendizaje (PLE) y factores clave de desarrollo curricular en el mundo digital.

E-mail: lindacq@um.es

Dirección:

Facultad de Educación

Universidad de Murcia

30100 Murcia (España)

Gema Tur. Doctora en Tecnología Educativa por la Universidad de las Islas Baleares, es profesora asociada del Departamento de Pedagogía Aplicada y Psicología de la Educación de la UIB, colabora en investigación con el Grupo de Infancia, Tencología, Educación y Diversidad, en el área de Tecnología Educativa,y coordina, entre otros, diversos programas de formación de profesores en la Sede Universitaria en Ibiza. Sus intereses de investigación incluyen eportfolios y Entornos Personales de Aprendizaje y social media, y en general, el aprendizaje enriquecido con tecnología en la formación docente inicial.

E-mail: gemma.tur@uib.es

Dirección:

Universitat de les Illes Balears

C/ del Calvari, 1

o780o Eivissa (España)

Ricardo Torres-Kompen. Profesor, Coordinador del Grado en International Computer Engineering. Investigador en el área de tecnologías del aprendizaje y profesor universitario desde 1996. Es ingeniero químico (1991), Master en Ingeniería Química (2000) y Doctor en Ingeniería Multimedia por la Universitat Politècnica de Catalunya- BarcelonaTech (2016). Su investigación se enfoca en la personalización del aprendizaje a través del uso de multimedia y tecnología.

E-mail: ricardot@salleurl.edu 
Dirección:

St Joan de La Salle 42, edif. Miquel Febres MF1.05

Barcelona 08022 (España)

Fecha de recepción del artículo: 18/05/2018

Fecha de aceptación del artículo: 29/06/2018

Fecha de aprobación para maquetación: 17/08/2018 